\title{
Quaderni
}

QUADERNI Communication, technologies, pouvoir

68 | Hiver 2008-2009

Militantisme médical et fabrique des politiques de santé

\section{Autisme et militantisme : de la maladie à la différence}

Autism and Advocacy: from the Disease to the Difference

\section{Brigitte Chamak}

\section{CpenEdition}

Journals

Édition électronique

URL : http://journals.openedition.org/quaderni/268

DOI : $10.4000 /$ quaderni.268

ISSN : 2105-2956

Éditeur

Les éditions de la Maison des sciences de l'Homme

Édition imprimée

Date de publication : 5 janvier 2009

Pagination : 61-70

Référence électronique

Brigitte Chamak, « Autisme et militantisme : de la maladie à la différence », Quaderni [En ligne] 68 | Hiver 2008-2009, mis en ligne le 05 janvier 2012, consulté le 30 avril 2019. URL : http:// journals.openedition.org/quaderni/268 ; DOI : 10.4000/quaderni.268 


\section{$D$ ossier}

\section{autisme et militantisme : de la maladie à la différence}

Dans la ligne de réflexion de Ian Hacking (2005) sur les classifications, j'ai cherché à comprendre comment les transformations de catégorie - ellesmêmes reflet de mutations sociétales - influencent les personnes concernées. Les changements de catégorie de l'autisme, initiés au début des années 1990 par les nouvelles classifications des maladies (internationale et américaine), ont transformé les représentations de l'autisme. La maladie rare, incurable, souvent associée à un retard mental, décrite en 1943 par Léo Kanner, est devenue un syndrome aux contours flous étiqueté «troubles envahissants du développement» (TED). L'augmentation du taux de prévalence et la mobilisation des associations de parents ont fait de l'autisme une question de santé publique (Chamak, 2008).

CESAMES - Centre de Recherche Psychotropes, Santé Mentale, Société Inserm, U611 ; CNRS, UMR 8136 ; Université Paris Descartes
Cette catégorie, qui inclut désormais des personnes qui peuvent s'exprimer mais qui présentent une maladresse dans leurs contacts avec autrui et des difficultés de compréhension des codes sociaux, permet à un plus grand nombre d'individus de se reconnaître dans cette description et de témoigner de leur expérience de l'autisme (Chamak, 2005a). Ces changements s'accompagnent de l'émergence d'un nouveau mouvement social, regroupant des personnes qui refusent d'endosser le statut de patient. Elles formulent de nouvelles revendications dans la mouvance de celles des associations de personnes handicapées (Driedger, 1989, Albrecht et al. 2001) et, plus largement, de celles des minorités - féministes, gays, noires, etc.

Les actions des associations de personnes autistes, moins médiatisées que celles des associations de parents, restent peu visibles, surtout 
en France. Pourtant, leur mobilisation commence à prendre de l'ampleur via Internet. La première association, Autism Network International (ANI), créée en 1991 aux États-Unis, défend l'idée selon laquelle l'autisme ne serait ni une maladie ni un handicap mais un autre mode de fonctionnement cognitif, une autre façon de penser, d'agir et de vivre. Cette référence à la différence constitue l'un des nombreux signes révélateurs d'une transformation plus profonde de notre société où les nouvelles normes de l'autonomie modifient l'image que les individus portent sur eux-mêmes et sur les autres (Ehrenberg, 1991).

Le mouvement des personnes autistes est beaucoup plus marginal en France que dans d'autres pays (États-Unis, Canada, Royaume-Uni, Australie...). Comment expliquer cette différence? D'une part, l'élargissement des critères diagnostiques de l'autisme a été beaucoup plus tardif en France (Chamak, 2008). D'autre part, la dynamique historique des associations françaises permet de mieux comprendre pourquoi les associations de personnes présentant un handicap ont du mal à faire entendre leurs voix face aux associations de parents qui ont acquis un quasimonopole grâce à leurs fonctions gestionnaires et à leur fort partenariat avec l'État (Barral et al. 2000, Barral 2007).

Cet article a pour objectif d'analyser les mouvements sociaux associés à l'autisme, de comparer la dynamique historique de la création de l'ANI avec celle de la mobilisation des personnes autistes en France, de préciser leur positionnement par rapport aux actions des associations de parents, mais aussi d'aborder la question de la spécificité du système associatif français.

\section{Dynamique historique de la mobilisation internationale des associations de personnes autistes}

L'association ANI est considérée, aujourd'hui, comme la première et la plus importante communauté regroupant des personnes autistes (Chamak, 2008). À l'initiative de Jim Sinclair, un premier groupe d'entraide s'est constitué au début des années 1980 réunissant des personnes qui, comme lui, découvraient que le qualificatif d'autisme correspondait le mieux à leur façon d'être et de vivre.

Des échanges via Internet sur un forum créé par une association de parents ont précédé des rencontres qui ont eu lieu lors de conférences sur l'autisme. Une correspondance par messages électroniques privés s'est développée, des rendezvous ont été pris et, en 1991, ce premier groupe de personnes autistes a participé à la conférence organisée par la société américaine pour l'autisme (Autism Society of America, ASA). Les organisateurs ont proposé à ce groupe de former un comité de réflexion qui, rapidement, perçut les comportements des dirigeants d'ASA comme paternalistes et irrespectueux. Les personnes autistes interprétèrent comme une instrumentalisation ce qu'ils avaient pris pour une réelle collaboration et décidèrent de fonder leur propre association. À peine ANI était-elle créée que des rumeurs en provenance d'ASA circulèrent sur Jim Sinclair interrogeant la réalité de son autisme dans l'intention de disqualifier ce nouveau mouvement qui prenait forme (Sinclair, 2005).

En novembre 1992 paraît le premier numéro de Our Voice. Cette revue favorise le partage 
d'informations tandis que le forum facilite les échanges individuels (Sinclair, 2005). La même année, Jim Sinclair s'exprimait dans l'ouvrage consacré aux personnes autistes présentant un haut niveau de fonctionnement dirigé par E. Schopler et G. Mesibov (1992) : «Accordezmoi la dignité de me rencontrer selon mes propres termes - reconnaître que nous sommes également étrangers l'un à l'autre, que ma façon d'être n'est pas simplement une version déficiente de la vôtre. Interrogez-vous sur vos présupposés. Travaillez avec moi à construire davantage de ponts entre nous $»^{1}$.

Entre 1993 et 1994, plusieurs événements contribuèrent à transformer un petit réseau en une véritable communauté (un groupe de personnes avec des intérêts communs et perçu comme un segment distinct de la société). La conférence internationale sur l'autisme en juin 1993 à Toronto a permis à Jim Sinclair de marquer un point important. Sa présentation en forme de message personnel, « Ne vous lamentez pas pour nous », a eu un fort impact. Il remettait en cause la description de l'autisme comme une tragédie, faite en particulier par les associations de parents : "L'autisme n'est ni quelque chose qu'une personne a, ni une "coquille» dans laquelle elle se trouve enfermée. Il n'y a pas d'enfant normal caché derrière l'autisme. L'autisme est une manière d'être. Il est envahissant; il teinte toute sensation, perception, pensée, émotion, tout aspect de la vie. Il n'est pas possible de séparer l'autisme de la personne - et si c'était possible, la personne qui resterait ne serait plus la même [...] Par conséquent, quand les parents disent : « je voudrais que mon enfant n'ait pas d'autisme » ce qu'ils disent vraiment c'est « je voudrais que l'enfant autiste que j'ai n'existe pas. Je voudrais avoir, à la place, un enfant différent (non autiste) ». C'est ce que nous entendons quand vous vous lamentez sur notre existence et que vous priez pour une guérison $»^{2}$.

Ce discours a rencontré un large écho et de nombreuses personnes autistes ont adhéré à ANI. Il a également suscité une augmentation des échanges via Internet sur le forum et le mécontentement de quelques parents, submergés par des messages qui ne les intéressaient pas. En 1994, le forum des personnes autistes était créé (Autism Network International listserv, ANIL). À partir de 1996, ils décidèrent d'organiser eux-mêmes leurs conférences. Dénommées Autreat, les rencontres entre personnes autistes ont débuté à la campagne, lieu leur permettant de s'isoler facilement en cas de besoin. Elles regroupaient aussi bien des personnes autistes qui parlaient que d'autres n'étant pas à même de s'exprimer. Des enfants avec leurs parents étaient également présents. Lors de ces rencontres, les thèmes majeurs abordés étaient: la nécessité d'envisager les aspects positifs de l'autisme, les possibilités de fonctionnement dans un monde « neurotypique» (non autiste), et la place du mouvement autiste dans celui, plus large, des personnes handicapées.

Pour ANI, il ne s'agit pas de trouver des causes ou des remèdes à l'autisme, mais de former les personnes autistes afin qu'elles puissent assurer elles-mêmes leur propre défense, de constituer une communauté, d'accroître la visibilité publique de leurs actions, de réduire la stigmatisation, d'identifier et de sensibiliser des alliés potentiels au sein de la population non autiste ${ }^{3}$. En 2004, 
Autreat réunissait à l'Université de Syracuse des participants qui venaient principalement des États-Unis et du Canada, mais aussi d'Australie, de Finlande, d'Israël, du Japon, de NouvelleZélande, de Norvège, et d'ailleurs.

Les premières autobiographies parues dans les années 1980 et 1990, en particulier celles de Temple Grandin (1986) et de Donna Williams (1992), ont largement participé à la construction d'une politique identitaire autour de l'autisme (Chamak, 2008). N'étant plus limitée aux enfants sans langage, cette étiquette, si stigmatisante jusque-là, devenait un étendard pour des personnes fières de revendiquer le qualificatif d'autisme et décidées à en changer les représentations, refusant la vision négative et pessimiste et les descriptions perçues comme insultantes que les professionnels, les médias ou les parents faisaient de l'autisme. L'idée relayée par Internet selon laquelle Albert Einstein, Glenn Gould et d'autres génies célèbres auraient présenté un syndrome d'Asperger, forme d'autisme sans déficience intellectuelle et sans délai d'apparition du langage, contribue à alimenter un sentiment de fierté et, parfois même de supériorité. Lorsqu'ils se comparent aux individus dits «normaux », certains se trouvent plus rationnels et objectifs que ces « neurotypiques » guidés par leurs émotions, leurs sympathies et leurs inimitiés.

Michelle Dawson, personne autiste vivant à Montréal, dénonce, sur son site $\mathrm{Web}^{4}$, le fait que les associations de parents leur ont confisqué la parole. Poursuivant des recherches en sciences cognitives sur l'autisme pour mettre en évidence les capacités et les preuves d'intelligence des personnes autistes, elle se positionne fermement contre les méthodes comportementales, intente des actions en justice contre la discrimination et milite pour que les pouvoirs publics tiennent compte de l'avis des personnes autistes. Ces actions rappellent les mouvements des années 1970, en France, qui questionnaient les politiques publiques conçues pour les personnes handicapées et non par elles (Turpin, 1990, 2000). Les revendications et les résultats obtenus par les groupes d'auto-supports (self-help groups) (Katz et Bender, 1976 ; WHO, 1983), l'Independant Living Movement (De Jong, 1979) et les mouvements des minorités (noires, féministes, gays, etc.) ont servi de modèle aux mouvements de personnes présentant un handicap. Si les mouvements des personnes handicapées ont été considérés comme la dernière génération des mouvements sociaux (Driedger, 1989; Albrecht et al., 2001), les actions des personnes autistes peuvent être envisagées comme la dernière génération des mouvements de personnes handicapées.

Michelle Dawson considère que «l'autisme n'est pas plus une maladie que ne l'est l'homosexualité ». Elle se réfère explicitement à l'histoire des actions des féministes, des homosexuels et de différents groupes ethniques ${ }^{5}$. Elle mentionne le fait que pendant longtemps l'homosexualité a été injustement considérée comme une maladie psychiatrique. Elle refuse le statut de malade ou de patient et se focalise sur ce qui dans la société empêche les personnes autistes de trouver leur place et donc sur les causes structurelles de la stigmatisation. La construction sociale de l'image dévalorisante du handicap a déjà été dénoncée par Simi Linton (1995). Michelle Dawson et Jim Sinclair insistent sur cette vision limitée qui pénètre notre langage et sert à perpétuer 
une marginalisation et une discrimination des personnes autistes.

Initié au début des années 1990, ce mouvement international induit des transformations perceptibles dans les livres publiés par des personnes autistes. Les premiers ouvrages constituent des témoignages personnels décrivant leur expérience particulière de l'autisme. Les écrits ultérieurs témoignent d'une volonté de participer à l'élaboration de véritables connaissances sur l'autisme et s'orientent vers une prise de conscience politique (Chamak, 2005a, 2008). L'usage du «nous » participe à la construction d'une communauté, avec une culture propre, des expressions et un humour spécifiques. Le sentiment d'une identité collective se développe, ainsi qu'une critique de la société où l'autisme est médicalisé et conçu sous le seul angle du modèle du déficit. L'identité se forge à travers la transformation des problèmes individuels en cause collective. Les personnes autistes qui s'expriment insistent sur l'importance de leur expérience pour comprendre leur situation et résoudre les problèmes rencontrés. Un nouveau courant, orienté vers un engagement direct et la légitimation des prises de parole des personnes autistes, s'est donc récemment constitué à l'échelle internationale.

\section{Mobilisation en France}

Ce mouvement de personnes autistes, qui tend à transformer des difficultés en connaissances, a émergé très récemment en France. La conscience d'appartenir à une communauté commence à se propager. L'association francophone Satedi s'est créée en 2002 - soit 11 ans après ANI pour apporter un éclairage sur le fonctionne- ment autistique, fournir de l'aide aux personnes autistes et à leur famille, et influer sur l'orientation de la recherche et les décisions politiques. Contrairement à ANI qui, d'emblée, s'était démarquée des associations de parents, Satedi est proche de certaines d'entre elles, et notamment d'Autisme France, créée en 1989 pour changer les interventions en autisme. Cette association de parents s'oppose résolument aux approches psychanalytiques de l'autisme, favorise le développement des méthodes éducatives et exige l'intégration scolaire. Avec le soutien d'autres associations, Autisme France a réussi à mobiliser les pouvoirs publics. Après la circulaire Veil du 27 avril 1995, proposant un plan d'action afin d'améliorer la prise en charge de l'autisme, la loi Chossy de 1996 définit l'autisme comme un handicap. Forte revendication des associations de parents, cette reconnaissance permet aux familles de bénéficier d'aides financières grâce à la loi d'orientation en faveur des personnes handicapées de 1975 (devenue la loi pour l'égalité des droits et des chances de 2005), de s'éloigner de la psychiatrie en rebaptisant handicap ce qui était considéré comme une maladie psychiatrique, de réclamer l'intégration scolaire - surtout depuis le vote de la loi sur l'égalité des droits et des chances de février 2005 qui pose le principe de l'inscription de tout enfant porteur de handicap dans l'établissement scolaire le plus proche du domicile (Chamak, 2005b, 2008).

En proposant à quelques membres de Satedi de faire partie de leur conseil d'administration, Autisme France obtenait la caution de personnes autistes tout en influençant leur façon d'analyser les problèmes liés à l'autisme et en rendant difficile pour Satedi de se démarquer des posi- 
tions d'Autisme France. La compréhension du contexte français permet de mieux appréhender cette difficulté d'émancipation. Contrairement aux États-Unis, au Canada, ou à d'autres pays européens, le mouvement social de revendication et d'action pour l'autonomie des personnes handicapées constitué autour de la notion de « civils rights » (Galli et Ravaud, 2000) est peu développé en France. L'essentiel des forces associatives est mobilisé par la gestion d'établissements assurée, majoritairement, par des associations de parents. Cette spécificité française consiste en de nombreuses institutions privées gérées par les associations qui les ont créées, sous tutelle du ministère de la Santé. Ce système de délégation de service public aux associations gestionnaires d'établissements spécialisés pour les populations handicapées a jeté les bases du partenariat entre l'État et les associations (Barral et al. 2000, Barral, 2007). Dès la fin de la Deuxième Guerre mondiale, des parents d'enfants handicapés se sont mobilisés pour pallier le manque de structures. Avec l'extension de la définition de l'inadaptation, les institutions se sont multipliées sur tout le territoire. L'élargissement des critères diagnostiques, qui ne concerne pas seulement l'autisme, accélère ce processus, donnant un poids toujours croissant aux associations gestionnaires, dans lesquelles parents et professionnels se partagent les responsabilités.

Les établissements restant sous contrôle du ministère de l'Action sociale et de son administration régionale et départementale, les associations doivent diversifier les modalités de négociations et les formes de pression aux différents échelons, nationaux et locaux, politiques et administratifs, pour consolider les liens avec l'État qui garantis- sent leur pérennisation. Catherine Barral (2007) a bien montré comment l'institutionnalisation des relations entre l'État et les associations prend parfois des formes personnalisées avec une circulation entre services de l'État et associations. De hauts fonctionnaires ou des élus rejoignent les conseils d'administration des associations et des administrateurs associatifs intègrent la haute fonction publique. Catherine Barral (2007) insiste sur le fait que les associations gestionnaires se sont vues confirmées dans leur partenariat avec l'État pour la poursuite d'une politique sociale qui répond davantage à des impératifs de gestion de populations qu'à ceux de réinsertion sociale.

Ce mode de fonctionnement permet de mieux comprendre les difficultés, en France, des associations de personnes autistes - et plus largement de personnes handicapées - à défendre leur propre point de vue et à exister en tant qu'organisations autonomes avec des revendications différentes de celles des associations de parents. Il explique aussi pourquoi le mouvement de vie autonome (Independant Living Movement), développé en Amérique du Nord, reste quasi inexistant en France. De même, les « disability studies », qui dénoncent le modèle médical du handicap et insistent sur l'origine sociale de la stigmatisation, demeurent réduites à la portion congrue alors qu'elles fleurissent aux États-Unis, au Canada et au Royaume-Uni. Le courant de chercheurs en sciences cognitives qui travaillent avec des personnes autistes sans déficience intellectuelle et qui font référence à la neurodiversité (BaronCohen, 2000 ; Happé, 1999 ; Mottron, 2004) vivent au Royaume-Uni et au Québec.

Les témoignages de personnes autistes sont très 
rares en France. Un seul livre, rédigé par un adolescent (Bouissac, 2002), a été publié. Il décrit son expérience individuelle avec ses intérêts, ses obsessions, ses difficultés, ses espoirs. Ce récit se distingue de ceux, plus politiques, publiés, à la même époque, dans d'autres pays. Il existe, pourtant, au sein de Satedi, des personnes qui commencent à élaborer des revendications différentes de celles des associations de parents.

\section{Perspectives}

Au moment où la classification américaine des maladies est en cours de révision pour préparer le DSM-V, il paraît judicieux d'analyser ce que les changements de classifications - et au-delà les transformations de nos sociétés qui s'y reflètent - impliquent pour les personnes concernées. Le classement dans une même catégorie d'enfants sans langage et de personnes présentant des capacités langagières et des difficultés d'interactions sociales a accru la prévalence de l'autisme et sa visibilité (Chamak, 2005b, 2008). De nouvelles associations se sont créées, leurs revendications ont pris davantage de poids, le problème n'était plus circonscrit mais érigé en question de santé publique (Chamak, 2008). Interpellés, les pouvoirs publics ont dû se mobiliser, des circulaires et des lois ont été votées. Les marchés de l'autisme se sont développés de façon exponentielle, les financements pour la recherche également, de nouveaux professionnels et chercheurs se sont spécialisés dans l'autisme. Des adultes qui se sentaient différents mais ne savaient pas en quoi consistait cette différence se sont reconnus dans la description du fonctionnement autistique. Ils ont d'abord témoigné de leur expérience en écrivant leur biographie. Ils ont ensuite tenté de changer l'image négative de l'autisme en montrant en quoi l'originalité et la créativité des personnes présentant des caractéristiques autistiques enrichissent une société. Ils ont produit un discours de type culturaliste qui célèbre la « culture autistique », mettant l'accent sur les aspects positifs et créateurs. Ainsi a émergé un nouveau mouvement social, articulé autour d'affiliations identitaires et culturelles, redéfinissant l'autisme comme une différence, et non comme une maladie, suivant en cela une logique d'émancipation du modèle biomédical (Chamak, 2008).

Ce mouvement a été plus important dans certains pays (États-Unis, Canada, Royaume-Uni, Australie...) que dans d'autres, et notamment en France, où le contexte culturel et l'histoire des associations sont bien différents. La spécificité française décrite par C. Barral (2007) - des associations gestionnaires d'établissements spécialisés qui jettent les bases du partenariat entre l'État et les associations (essentiellement de parents) - permet de mieux comprendre le quasimonopole de ces associations à la tête de plus de $80 \%$ des établissements et services. Ce modèle de l'association gestionnaire par délégation de service public laisse peu de place aux associations de personnes présentant un handicap.

Dans les années 1970, les mouvements contestataires d'étudiants handicapés ont porté, en France, la revendication d'autonomie et d'exercice des droits civils des associations d'usagers (Turpin, 2000). Marginalisés par «l'establishment associatif » et par l'échec des tentatives d'alliances avec d'autres mouvements sociaux, ils ont disparu au cours de la décennie 1980. Leur analyse critique des causes socio-politiques et structu- 
relles du handicap mettait en cause le modèle de la réadaptation, le partenariat entre l'État et « l'establishment associatif» et le sens général des politiques sociales qui administrent la vie personnelle, sociale et professionnelle des personnes handicapées. Ils dénonçaient la collusion entre associations gestionnaires et pouvoirs publics. Comme le font aujourd'hui certaines associations de personnes autistes, ils reprochaient aux associations dominantes, contrôlées par des parents et des professionnels, leur paternalisme vis-à-vis des personnes handicapées. L'identité des personnes autistes se construit actuellement dans le cadre d'une résistance à la définition de l'autisme comme une maladie et dans la volonté de faire entendre leur voix. Cette conscience politique est moins développée en France mais un changement s'opère peut-être. Sous la pression des politiques publiques européennes, la France a introduit, dans la loi du 11 février 2005, la possibilité d'aide à la mise en place de groupes d'entraide mutuelle (GEM), perçus comme un moyen de réinsérer dans la cité les personnes concernées par les troubles psychiques. Les programmes d'action initiés par les Nations Unies et la Commission européenne participeront-ils à une plus grande visibilité des mouvements de personnes présentant un handicap en France? Les tentatives des grandes associations gestionnaires pour obtenir des financements afin de créer des GEM ne vont pas forcément dans ce sens. Il existe, toutefois, des « espaces conviviaux citoyens » créés par Advocacy France qui, eux, agissent comme des GEM à part entière.

Remerciements : Je tiens à remercier Béatrice Bonniau pour son aide précieuse et Laurent Lefèvre pour sa lecture attentive et ses corrections judicieuses.

\section{$R \cdot E ́ \cdot F \cdot E ́(R \cdot E \cdot N \cdot C \cdot E \cdot S$}

G. ALBRECHT, J-F.RAVAUD, H-J. STIKER

«L'émergence des disabilities studies : état des lieux et perspectives », Sciences sociales et Santé, 2001, n 19, pp. 43-76.

S. BARON-COHEN, « Is Asperger's syndrome/ high-functioning autism necessarily a disability ? » Development and Psychopathology 2000, 12 : 489-500.

C. BARRAL, F. PATERSON, H-J. STIKER, M. CHAUVIÈRE, L'institution du handicap : le rôle des associations, Rennes, Presses Universitaires de Rennes, 2000.

C. BARRAL, « Disabled Persons’ Associations in France ", in Scandinavia Journal of Disability Research, vol. 9, n 3-4, 2007, pp. 214-236.

J. BOUISSAC, Qui j'aurai été..., Autisme Alsace, 2002.

B. CHAMAK, «Les récits de personnes autistes : une analyse socio-anthropologique », in handicap, revue de sciences humaines et sociales, $\mathrm{n}^{\circ}$ 105-106, 2005a, pp. 33-50.

B. CHAMAK, « Les transformations des représentations de l'autisme et de sa prise en charge en France : le rôle des associations ", in Nouveau malaise dans la civilisation, Cahiers de Recherche Sociologique, 41, 2005b, pp.171-192.

B. CHAMAK, « Autism and Social Movements : French Parents' Associations and International Autistic Individuals' Organizations », in Sociology of Health and Illness, vol.30, n 1, 2008, pp. 76-96. G. De JONG, «Independant Living, from social to analytic paradigm ", Archives of Physical Medecine and Rehabilitation, $\mathrm{n}^{\circ}$ 60, 1979, pp. 435-446.

D. DRIEDGER, The Last Civil Rights Movement, 
London, Hurst and Co, 1989.

A. EHRENBERG, Le culte de la performance, Calmann-Lévy, 1991.

F. HAPPÉ, « Autism: cognitive deficit or cognitive style? » Trends in Cognitive Sciences 1999, 3 (6): 216-222.

C. GALLI, J.F. RAVAUD, « L'association vivre debout: une histoire d'autogestion », in L'institution du handicap : le rôle des associations, Rennes, Presses Universitaires de Rennes, 2000.

I. HACKING, « Façonner les gens », Cours au Collège de France, 2005.

A.H. KATZ, E.L. BENDER, « Self-help groups in Western society : history and prospects ", $J$. Appl. Behav. Sci., n 12, 1976, pp. 265-282.

S. LINTON, Claiming Disability: Knowledge and Identity, New York: New York University Press, 1995.

L. MOTTRON, L'autisme: une autre intelligence. Diagnostic, cognition et support des personnes autistes sans déficience intellectuelle, Belgique : Mardaga, 2000.

E. SCHOPLER, G. MESIBOV, High-functioning Individuals with Autism, Plenum Press, New York, 1992, pp. 294-302.

J. SINCLAIR, « Autism network international: the development of a community and its culture », 2005. http://web.syr.edu/ jisincla/History_of_ANI.html

P. TURPIN, « La lutte contre l'assistance pendant les années soixante-dix », Cahiers du CTNERHI, $\mathrm{n}^{\circ}$ 50, 1990, pp. 83-92.

P. TURPIN, « Les mouvements radicaux de personnes handicapées en France pendant les années $1970 »$, in L'institution du handicap : le rôle des associations, Rennes, Presses Universitaires de Rennes, 2000.

WHO, Self-help and health in Europe, 1983.
$\mathrm{N} \cdot \mathrm{O} \cdot \mathrm{T} \cdot \mathrm{E} \cdot \mathrm{S}$

1. J. Sinclair, « Bridging the gaps: an inside-out view of autism (or, do you know what I don't know?) » in E. Schopler and G. Mesibov, High-functioning Individuals with Autism, Plenum Press, New York, 1992, p. 302. « Grant me the dignity of meeting me on my own terms - recognize that we are equally alien to each other, that my ways of being are not merely damaged versions of yours. Question your assumptions. Define your terms. Work with me to build more bridges between us ».

2. J. Sinclair, Don't mourn for us, Our Voice, the newsletter of Autism Network International 1, 1993 - www.jimsinclair.org/dontmourn.htm.

"Autism isn't something a person has, or a "shell" that a person is trapped inside. There's no normal child hidden behind the autism. Autism is a way of being. It is pervasive; it colors every experience, every sensation, perception, thought, emotion, and encounter, every aspect of existence. It is not possible to separate the autism from the person - and if it were possible, the person you'd have left would not be the same person you started with. ... Therefore, when parents say, "I wish my child did not have autism", what they're really saying is, "I wish the autistic child I have did not exist, and I had a different (non-autistic) child instead. This is what we hear when you mourn over our existence. This is what we heard when you pray for a cure $»$.

3. Cf. le site d'ANI: http://ani.autistics.org/

4. http://www.sentex.net/ nexus23/naa_aba.html

5. Entretien avec Michelle Dawson à Montréal le 10 août 2004. 
Au début des années 1990, la catégorie « autisme » a été modifiée. La maladie rare décrite par Leo Kanner en 1943 est devenue un syndrome aux contours flous étiqueté « troubles envahissants du développement ». En incluant dans cette catégorie des individus qui peuvent s'exprimer mais qui présentent des difficultés d'interactions sociales, cette classification permet à de nombreuses personnes de se reconnaître dans cette description. Ces transformations coïncident avec l'émergence d'un nouveau mouvement social qui critique une société où l'autisme est conçu sous le seul angle du modèle du déficit. La première association, Autism Network International, créée en 1991 aux ÉtatsUnis, défend l'idée selon laquelle l'autisme ne serait ni une maladie ni un handicap mais un autre mode de fonctionnement cognitif, une différence. Cette concep- 